\title{
Curved trajectories on transformed metal surfaces: Luneburg lens, beam-splitter, invisibility carpet and black hole for surface plasmon polaritons.
}

\author{
Kadic Muamer*, Dupont Guillaume, Tieh-Ming Chang, Sebastien Guenneau, \\ Stefan Enoch \\ Institut Fresnel, CNRS, Aix-Marseille Université, \\ Campus universitaire de Saint-Jérôme, 13013 Marseille, France
}

\begin{abstract}
Transformational optics are shown to markedly enhance the control of the electromagnetic wave trajectories within metamaterials with unconventional functionalities such as a beam splitter, a toroidal carpet, a Luneburg lens and a black hole, all of which are specially designed for surface plasmon polaritons propagating on a metal plate.
\end{abstract}

Keywords: Surface plasmon polariton, transformational plasmonics, transformational optics, Luneburg lens, beam splitter, black hole, carpet

\section{Introduction}

In 1998, a team led by Ebbesen discovered that resonant excitations of surface plasmons enhance electric fields at a surface that force light through its tiny holes, giving very high transmission coefficients in the sub-wavelength regime [1]. Five years afterwards, Pendry, Martin-Moreno and Garcia-Vidal subsequently proposed a homogenized model of such structured metal surfaces in order to push the dispersion relation of surface plasmon polaritons to new boundaries [2]. Plasmonics actually holds new promises with recent proposals in invisibility cloaks relying upon plasmonic metamaterials that have already led to fascinating results [3, 4, 5, 6]. These include resonant shells with a suitable out-of-phase polarizabil-

\footnotetext{
*Corresponding author

Email address: muamer.kadicafresnel.fr (Kadic Muamer)
} 
ity in order to compensate the scattering from the knowledge of the electromagnetic parameters of the object to hide, and external cloaking, whereby a plasmonic resonance cancels the external field at the location of a set of electric dipoles. Recently, Baumeier et al. have demonstrated theoretically and experimentally that it is possible to reduce significantly the scattering of an object by a surface plasmon polariton, when it is surrounded by two concentric rings of point scatterers [6]. In this invited paper, we propose three plasmonic devices designed using powerful tools of transformational optics. We validate our theoretical proposals with three-dimensional computations using the finite element package COMSOL MULTIPHYSICS. This emerging area of photonics is fueled by analogies with cosmologic physics in non-Euclidean space-time metrics [7]: here, we focus our attention on a beam splitter, a toroidal carpet, a Luneburg lens and an optical black hole, for surface plasmons propagating at an anisotropic metal-dielectric interface. These four electromagnetic paradigms emphasize an unprecedented control of surface waves using mathematical tools of general relativity [8, 9] in order to design some meta-surfaces [10]-[15] achieving new plasmonic functionalities.

\section{Beam splitter}

Recent advances in transformational optics have led to the proposal by de Rham et al. of a beam splitter which is a heterogeneous anisotropic two-dimensional slab splitting an incident beam into two beams propagating along different directions. Importantly, the slab is impedanced matched to the surrounding medium, hence it does not exhibit any reflection at the entrance or exit boundaries [16]. We would like to adapt this device to the case of surface plasmon polaritons propagating at a transformed metal-dielectric interface. The bottom-neck of the design is the following transform [16]:

$$
\left\{\begin{array}{l}
x^{\prime}=x \\
y^{\prime}=\frac{y_{2}-y_{1}}{y_{2}} y+y_{1} \\
z^{\prime}=z
\end{array} \quad \Rightarrow \quad \mathbf{J}_{r r^{\prime}}=\left(\begin{array}{ccc}
1 & 0 & 0 \\
c_{21} & \alpha^{-1} & 0 \\
0 & 0 & 1
\end{array}\right)\right.
$$

where $\mathbf{J}_{r r^{\prime}}$ is the Jacobian matrix of the transformation, and $c_{12}$ is given by :

$$
c_{21}=y_{2} \frac{y-y_{2}}{\left(y_{2}-y_{1}\right)^{2}} \frac{\partial y_{1}}{\partial x^{\prime}}
$$

The inverse transformation matrix takes the following form: 

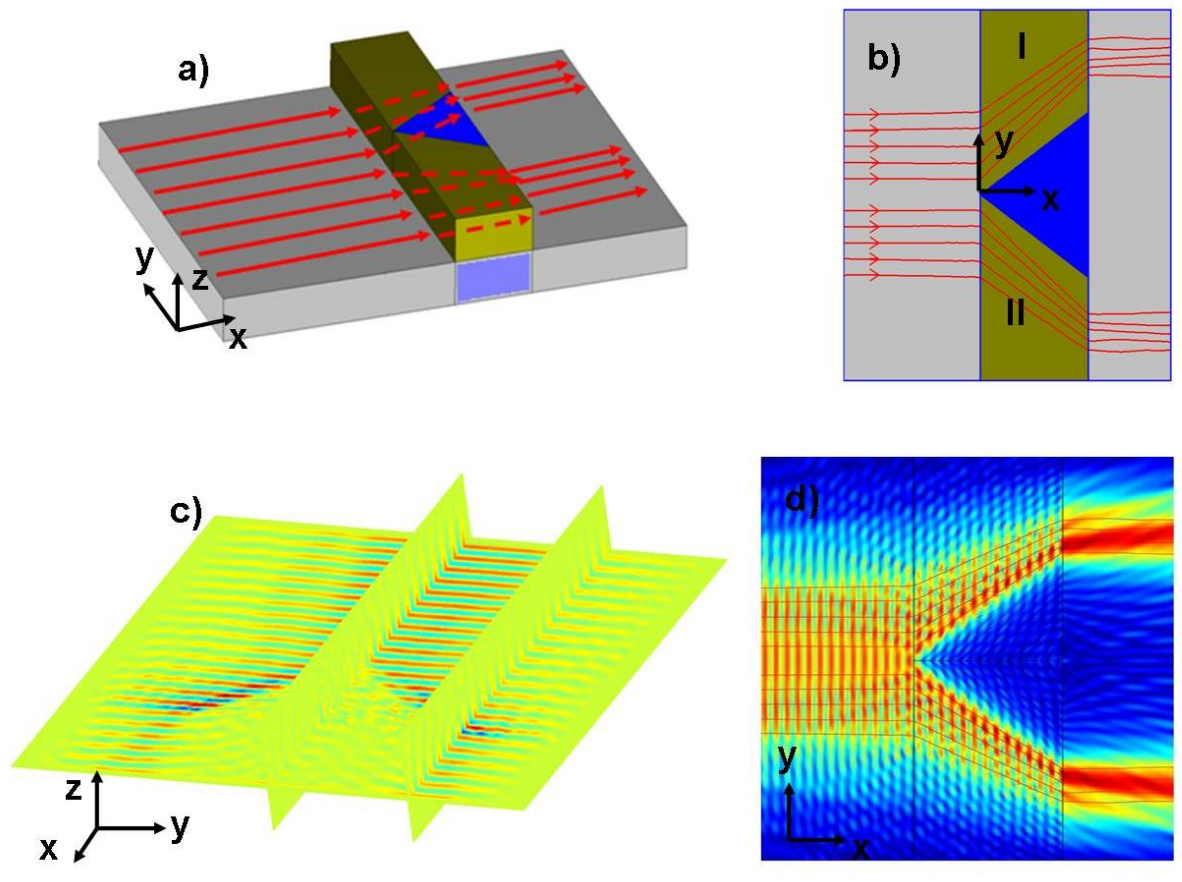

Figure 1: Beam splitter, a) Schematic diagram for a SPP beam splitter. A SPP is propagating from the left side. b) Top view of beam splitter with regions I and II deduced from the geometric transform 1 with $y_{1}=x, y_{2}=1$ in I and $y_{1}=-x$, $y_{2}=-1$ in II; c) Three-dimensional plot of the real part of the magnetic field for a $y$-polarised SPP propagating in $x$-direction (phase); d) Two-dimensional plot of the normalised powerflow of the magnetic field in the $(x, y)$ plane. Stream lines appear in red color.

$$
\mathbf{T}^{-1}=\mathbf{J}_{x x^{\prime}}^{-1} \mathbf{J}_{x x^{\prime}}^{-T} \operatorname{det}\left(\mathbf{J}_{x x^{\prime}}\right)
$$

where $\mathbf{J}_{x x^{\prime}}^{-T}$ denotes the inverse transpose matrix of $\mathbf{J}_{x x^{\prime}}$, and $\operatorname{det}\left(\mathbf{J}_{x x^{\prime}}\right)$ its determinent. Besides, the explicit expression of $\mathbf{T}^{-1}$ is:

$$
\mathbf{T}^{-1}=\left(\begin{array}{ccc}
\alpha^{-1} & -c_{21} & 0 \\
-c_{21} & \alpha\left(1+c_{21}^{2}\right) & 0 \\
0 & 0 & \alpha^{-1}
\end{array}\right)
$$

where $\alpha=\frac{y_{2}-y_{1}}{y_{2}}$. We show in figure 1 a typical computation for a beam splitter 
in the particular case when the functions $y_{1}$ and $y_{2}$ bounding the regions I and II are straight lines symmetric with respect to $y=0$ (here, $y_{1}= \pm x$ and $y_{2}= \pm 1$. We however note that $y_{1}$ and $y_{2}$ could any smooth functions.

\section{Toroidal Carpet}

Next, we propose a design of a toroidal carpet which is deduced from the following transform:

$$
\left\{\begin{array}{l}
x^{\prime}=x \\
y^{\prime}=y \\
z^{\prime}=\frac{z_{2}-z_{1}}{z_{2}} z+z_{1}
\end{array}\right.
$$

where $\mathbf{J}_{z z^{\prime}}$ is the Jacobian matrix of the transformation as per:

$$
\mathbf{J}_{z z^{\prime}}=\left(\begin{array}{ccc}
1 & 0 & 0 \\
0 & 1 & 0 \\
\frac{\partial z}{\partial x^{\prime}} & \frac{\partial z}{\partial y^{\prime}} & \alpha^{-1}
\end{array}\right)
$$

and partial derivatives can be expressed as:

$$
\frac{\partial z}{\partial x^{\prime}}=z_{2} \frac{z^{\prime}-z_{2}}{\left(z_{2}-z_{1}\right)^{2}} \frac{\partial z_{1}}{\partial x^{\prime}}+z_{1} \frac{z_{1}-z^{\prime}}{\left(z_{2}-z_{1}\right)^{2}} \frac{\partial z_{2}}{\partial x^{\prime}} \quad ; \quad \frac{\partial z}{\partial y^{\prime}}=z_{2} \frac{z^{\prime}-z_{2}}{\left(z_{2}-z_{1}\right)^{2}} \frac{\partial z_{1}}{\partial y^{\prime}}+z_{1} \frac{z_{1}-z^{\prime}}{\left(z_{2}-z_{1}\right)^{2}} \frac{\partial z_{2}}{\partial y^{\prime}}
$$

The resulting transformation matrix is:

$$
\mathbf{T}^{-1}=\left(\begin{array}{ccc}
\alpha^{-1} & 0 & -\frac{\partial z}{\partial x^{\prime}} \\
0 & \alpha^{-1} & -\frac{\partial z}{\partial y^{\prime}} \\
-\frac{\partial z}{\partial x^{\prime}} & -\frac{\partial z}{\partial y^{\prime}} & \alpha\left(1+\left(\frac{\partial z}{\partial x^{\prime}}\right)^{2}+\left(\frac{\partial z}{\partial y^{\prime}}\right)^{2}\right)
\end{array}\right)
$$

In the design shown in figure 2, we actually mapped a toroidal bump (described by the surface of altitude $z_{1}$ ) on a flat ring (described by the surface $z_{2}$ ):

$$
\begin{aligned}
& z_{1}=\sqrt{b^{2}-\left(\sqrt{x^{2}+y^{2}}-a\right)^{2}}+z o \quad \text { with } \quad a=0.3 ; b=0.1 ; z o=-0.05 \\
& z_{2}=0.2
\end{aligned}
$$




$$
\frac{\partial z_{1}}{\partial x}=-\frac{\left(\sqrt{x^{2}+y^{2}}-a\right) x}{\sqrt{b^{2}-\left(\sqrt{x^{2}+y^{2}}-a\right)^{2}} \cdot \sqrt{x^{2}+y^{2}}} \quad ; \quad \frac{\partial z_{1}}{\partial y}=-\frac{\left(\sqrt{x^{2}+y^{2}}-a\right) y}{\sqrt{b^{2}-\left(\sqrt{x^{2}+y^{2}}-a\right)^{2}} \cdot \sqrt{x^{2}+y^{2}}}
$$

We show in figure 2 the result of our three-dimensional computations, which clearly demonstrate that an electromagnetic field can be stored within an invisible carpet that does not perturb the ambient SPP field. Applications might be in harddiscs for an all-optic computer.

a)
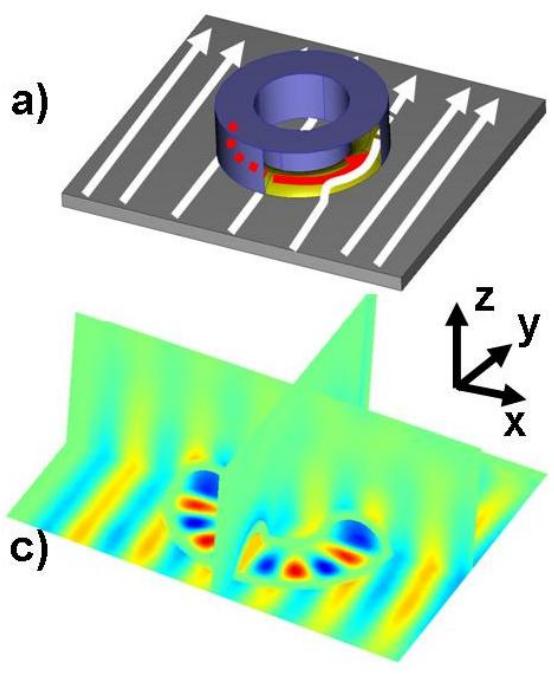
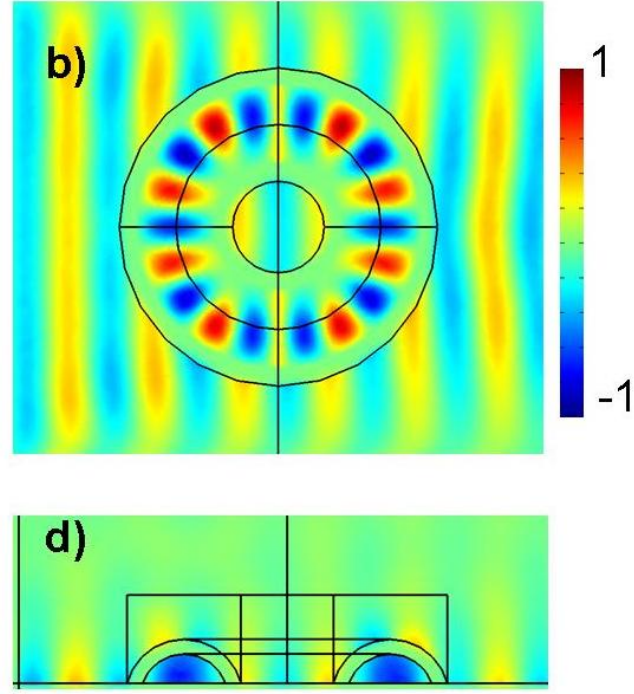

Figure 2: Plane wave, a) Electromagnetic field incident from the top (z-axis), with the magnitude of the magnetic field being represented; b) Phase representation of the magnetic field. $y$-polarized SPP wave; c) Two-dimensional plot in the $(x, y)$ plane; d) Two-dimensional plot in the $(x, y)$ plane; Moreover, an electromagnetic field has been launched inside the toroidal carpet filled here with

a dielectric medium of permittivitty $\varepsilon=2$ and surrounded by a thin metal coating. The field circulates in closed trajectories and does not perturb the SPP propagation on the metal plate. 


\section{Luneburg lens}

There is currently a renewed interest in gradient index lenses, such as Eaton and Luneburg lenses and Maxwell's fisheye. Following the earlier proposal by Liu et al. [12] of a Luneburg lens which is made of a homogeneous dielectric with a specific spatial variation of the profile, we decide here to investigate the case of a heterogeneous Luneburg lens, with the usual spatially varying refractive index given by [18]:

$$
n=\sqrt{2-\left(\sqrt{x^{2}+y^{2}} / R\right)^{2}},
$$

where $R$ is the radius of the lens and the coordinate axis is located at the center of the lens.
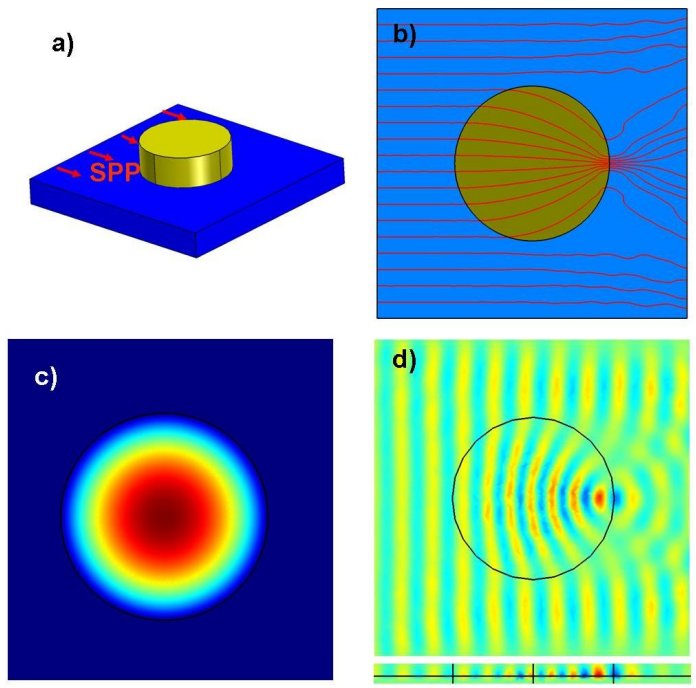

Figure 3: Plasmonic cylindrical Luneburg lens with a spatially varying refractive index. (a) Schematic diagram for a cylindrical Luneburg lens on a metal plate

(the color scale ranges from 1 (vacuum), blue color, to 1.414, red color; (b)

Streamlines show the focussing effect for a SPP incident upon the Luneburg lens (view from above); (c) Spatial distribution of the refractive index; (d) Top view and side view for a $y$-polarized SPP propagating from the left and incident upon the Luneberg lens at $700 \mathrm{~nm}$ (2D plots of the normalized real part of the magnetic field in the $(x, y)$ and $(x, z)$ planes).

We note that the metal is kept untounched, and no magnetism is involved in 
this design which thus looks as a reasonable design for an experimental vailidation.

\section{Black hole}
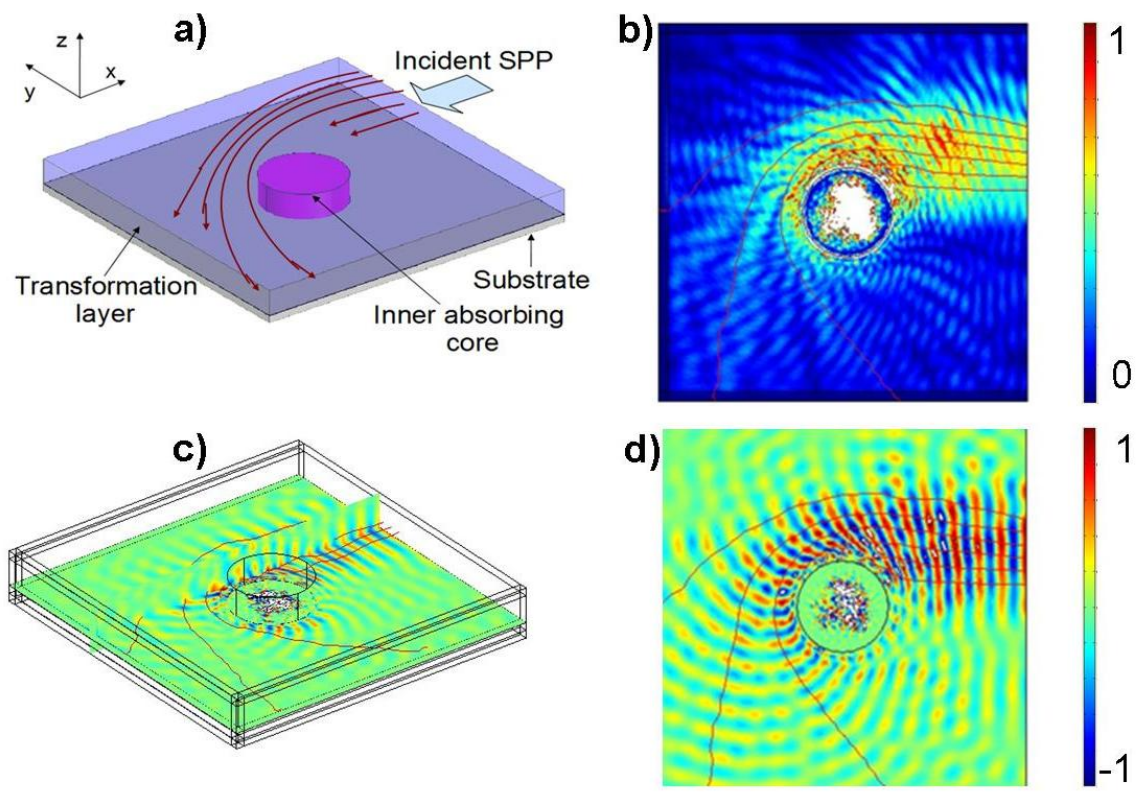

Figure 4: Plasmonic cylindrical black hole mimicking a Schwarschild metric. (a) Schematic diagram for a transformed metal plate with a cylindrical transformed medium on the top; (b,c,d) A SPP Gaussian beam making an angle of 60 degrees with the $x$-axis is incident from the right (b: top view, normalized magnitude of magnetic field; c: 3D plot for the normalized real part of magnetic field; d: top view phase of magnetic field).

The infinitesimal line element in a Schwarschild metric for a black hole can be expressed as [8]

$$
\left.d s^{2}=\left(1-\frac{L}{r}\right) c^{2} d t^{2}\right)-\left(1-\frac{L}{r}\right)^{-1} d r^{2}-r^{2} d \theta^{2}-d z^{2},
$$

where $L$ denotes the event horizon [7], $t$ is the time variable, $(r, \theta, z)$ denote the space coordinates and $c$ is the velocity of light. Without loss of generality, one can 
simply skip the time variable within the framework of time-harmonic Maxwell's equations.

Following the proposal of an optical black hole by Chen et al. [19], we rederived the transformation matrix associated with the spatial part of the Schwarschild metric and we found that

$$
\mathbf{T}^{-1}=\frac{A}{1-\frac{L_{1}}{r}}\left(\begin{array}{ccc}
1-\frac{L_{x}^{2}}{r^{3}} & -\frac{L_{x y}}{r^{3}} & 0 \\
-\frac{L_{x y}}{r^{3}} & 1-\frac{L_{y}^{2}}{r^{3}} & 0 \\
0 & 0 & 1
\end{array}\right)
$$

where $A=1-i$ in the absorbing core $(r \leq L)$ and $A=1$ in the transformation layer $(r>L)$.

In the present case, we launch a SPP at the wavelength $\lambda=700 \mathrm{~nm}$ and width $1400 \mathrm{~nm}$. The radius of the inner absorbing core is $L=0.9 \mu \mathrm{m}$ and $L_{1}=1 \mu \mathrm{m}$. In figure 4 , the trajectory of the Gaussian beam is clearly bent around the center part of the black hole (see also the streamlines), as it should in a Schwarschild metric.

\section{Conclusion}

In this paper, we reviewed some techniques of transformational optics applied to surface plasmon polaritons propagating at a flat interface separating a transformed Drude metal and a transformed dielectric medium. We have proposed four original meta-surfaces: A beam splitter which splits a large Gaussian SPP in two narrower Gaussian SPPs with different directions; A toroidal carpet which detours an SPP without disturbing its wavefront and its amplitude; A Luneburg lens which focusses an incident SPP onto a small region behind the lens; An optical black hole which can be modeled as vacuum solutions to the Einstein field equations. The latter device can be seen as intrinsic parts of the unbounded version of the Schwarzschild metric describing an eternal black hole with no charge and no rotation. Indeed, all geodesics of a free-falling particle in the spacetime can be continued arbitrarily far into the particle's future or past, unless the geodesic hits a gravitational singularity like the one at the center of the black hole's interior. In a similar way, we have seen that ray trajectories of a time-harmonic solution to the Maxwell's equations in the optical black hole converge towards the center of the black hole, in some kind of spiral manner, provided the rays intersect the event horizon of the black hole. 
We discovered while finalising the mansucript that a paper had been submitted by Zhang's team on numerical and experimental results for a plasmonic Luneberg lens, but we emphasize that our results cross-check their simulations.

\section{References}

[1] T. W. Ebbesen, H. J. Lezec, H. F. Ghaemi, T. Thio, P. A. Woff, Nature 391, 667 (1998).

[2] J.B. Pendry, L. Martin-Moreno and F.J. Garcia-Vidal, Mimicking surface plasmons with structured surfaces, Science 305, 847 (2004)

[3] N.A. Nicorovici, R.C. McPhedran and G.W. Milton, “Optical and dielectric properties of partially resonant composites," Phys. Rev. B 49, 8479-8482 (1994).

[4] A. Alu and N. Engheta, "Achieving transparency with plasmonic and metamaterial coatings," Phys. Rev. E 72016623 (2005).

[5] F.J. Garcia de Abajo, G. Gomez-Santos, L.A. Blanco, A.G. Borisov and S.V. Shabanov, Physical Review Letters 95067403 (2005).

[6] B. Baumeier, T.A. Leskova and A.A. Maradudin, "Cloaking from surface plasmon polaritons by a circular array of point scatterers," Physical Review Letters 103, 246809 (2009)

[7] S. Hawking and G. Ellis, The Large Scale Structure of Space-Time, Cambridge University Press (1973)

[8] J.A. Wheeler, Geometrodynamics. New York: Academic Press (1962)

[9] U. Leonhardt and T.G Philbin, "General relativity in electrical engineering," New J. Phys. 8247 (2006)

[10] I.I. Smolyaninov, "Transformational optics of plasmonic metamaterials," New J. Phys. 10(11), 115033 (2008).

[11] P.A. Huidobro, M. L. Nesterov, L. Martin-Moreno, and F. J. GarcaVidal,"Transformation Optics for Plasmonics," Nano Lett. 19, 1985 (2010).

[12] Y. Liu, T. Zentgraf, G. Bartal, and X. Zhang, "Transformational Plasmon Optics," Nano Lett. 6, 19911997 (2010). 
[13] I.I. Smolyaninov, V. N. Smolyaninova, A. V. Kildishev, and V. M. Shalaev, "Anisotropic metamaterials emulated by tapered waveguides: application to optical cloaking," Phys. Rev. Lett. 102(21), 213901 (2009).

[14] M. Kadic, S. Guenneau, and S. Enoch, "Transformational plasmonics: cloak, concentrator and rotator for SPPs," Opt. Express 18(11), 12027-12032 (2010).

[15] J. Renger, M. Kadic, G. Dupont, S.S. Acimovic, S. Guenneau, R. Quidant, and S. Enoch, "Hidden progress: broadband plasmonic invisibility," Optics Express 18(15), 15757-15768 (2010)

[16] M. Rahm, D.A. Roberts, J.B. Pendry, and D.R. Smith, "Transformationoptical design of adaptive beam bends and beam expanders," Optics Express 16(15), 11555-11567 (2008)

[17] M. Bashevoy, V. Fedotov, and N. Zheludev, "Optical whirlpool on an absorbing metallic nanoparticle,” Opt. Express 13, 8372-8379 (2005)

[18] R.K. Luneburg, "Mathematical Theory of Optics", Providence, Rhode Island: Brown University, 189213 (1944).

[19] H. Chen, R-X Miao and M. Li, Transformation optics that mimics the system oustide a Schwarzschild black hole, Opt. Express 15183-15188 (2010).

[20] T. Zentgraf, Y. Liu, M.H. Mikkelsen, J. Valentine and X. Zhang, Plasmonic Luneburg and Eaton lenses, Nature Nanotechnology (DOI: 10.1038/NNANO.2010.282) 